\title{
Anticholinerge Therapie der Reizblase: mehr Wirkung, mehr Nebenwirkungen?
}

— Die Prävalenz der hyperaktiven Blase ist mit $20 \%$ fast zehnmal so hoch wie jene von Diabetes und damit ein Thema, das sehr viele Patientinnen und Ärzte betrifft. Eine Reizblase kann die Lebensqualität der Patientin erheblich einschränken. Die Effizienz der verschiedenen Anticholinergika ist mit $60-80 \%$ sehr hoch, hoch ist aber auch der Placeboeffekt mit bis zu $40 \%$.

Für die Behandlung der Reizblase steht eine Vielzahl von Anticholinergika zur Verfügung. PD Dr. Annette Kuhn, Bern, Schweiz, präsentierte die Ergebnisse eines ICS-Workshops, in dem 2012 die Vor- und Nachteile der verschiedenen Substanzen evidenzbasiert beleuchtet wurden. „Ich glaube nicht, dass alle Anticholinergika gleich wirken", so Kuhn (vgl. Tabelle). Bei den meisten Anticholinergika treten bei höherer Dosierung verstärkt anticholinerge Nebenwirkungen, wie Mundtrockenheit, Schwindel, gastrointestinale Symptome und Sehstörungen auf, die zum Therapieabbruch führen können.

Für Patientinnen mit sehr starken Reizblasenbeschwerden, die auf Oxybutinin gut angesprochen, aber die Nebenwirkungen nicht toleriert haben, sei laut Kuhn Oxybutinin intravesikal eine erfolgversprechende Alternative.

„Mit den Anticholinergika dürfen wir eine moderate Verbesserung der Lebensqualität erwarten, aber keine $100 \%$ ige. Mit der Effi- zienz und der Tolerabilität stehen wir mit unseren derzeitigen Therapiemöglichkeiten ganz gut da", so die Einschätzung von Kuhn. Ulrike Tietze

A. Kuhn. Anticholinerge Therapie der Reizblase, 27.4.2013

\begin{tabular}{|c|c|c|}
\hline \multicolumn{3}{|c|}{ Vor- und Nachteile der verschiedenen Anticholinergika } \\
\hline Medikament & Vorteil & Nachteil \\
\hline Solifenacin & $\begin{array}{l}\text { Dosistitration } \\
\text { sehr gutes Wirkungs-NW-Profil }\end{array}$ & $\begin{array}{l}\text { trockener Mund bei } 10 \mathrm{mg} \\
\text { möglich }\end{array}$ \\
\hline Tolterodin & Einmaldosis, gute Datenlage & keine Dosistitration \\
\hline Fesoterodin & titrierbar & bei höherer Dosis mehr NW \\
\hline Oxybutinin & $\begin{array}{l}\text { billig } \\
\text { Daten in Schwangerschaft und } \\
\text { Laktation }\end{array}$ & $\begin{array}{l}\text { trockener Mund } \\
\text { kognitive Einschränkung } \\
\text { möglich }\end{array}$ \\
\hline Oxybutinin TD & konstante Plasmawerte & Hautirritation möglich \\
\hline Darifenacin & $\begin{array}{l}\text { kein Einfluss auf die kognitive } \\
\text { Funktion }\end{array}$ & gelegentlich Verstopfung \\
\hline Trospiumchlorid & $\begin{array}{l}\text { kein Überschreiten der } \\
\text { Blut-Hirn-Schranke } \\
\text { alleinige renale Elimination }\end{array}$ & $\begin{array}{l}\text { nicht auftitrierbar } \\
\text { zwei Dosen }\end{array}$ \\
\hline
\end{tabular}

\section{Überaktive Blase im Alter: Vorsicht mit Anticholinergika!}

— Das Gehirn steuert die Funktionen der Blase und ist für die Kontrolle der Kontinenz und das Anhalten der gefüllten Blase verantwortlich. Ein Großteil des Gehirns wird von Acetylcholin bedient, das hilft, Gedächtnisinhalte zu behalten. Im Alter nimmt das Acetylcholin im Gehirn ab und gleichzeitig lässt die Hirnleistung nach. Anticholinergika können Gedächtnisstörungen im Alter noch verstärken. Acetylcholin steuert viele Funktionen des Schlafes und wird entsprechend zirkadianer und ultradianer Schlafrhythmen freigesetzt. Acetylcholin vermittelt unter anderem die synaptische Plastizität und das Gedächtnis. „Im Schlaf werden die Lerninhalte in die Hirnrinde befördert. Bei gestörten Schlafphasen wird dieses Wissen nicht kristallisiert. Die Schlafarchitektur ist nur unbelastet, wenn man keine cholinergen Effekte zulässt", erläuterte Prof. Klaus-Christian Steinwachs, Nürnberg. Besonders bei den 1,2 Millionen De- menzkranken in Deutschland spielt das eine Rolle.

Bei Demenz liegt bereits ein Acetylcholinmangel vor. Anticholinergika können diesen Mangel bei Demenz verstärken und machen gleichzeitig eine antidementive Therapie wirkungslos. „Wir können die Acetylcholinwirkung nicht verstärken. Aber durch Acetylcholinesterase-Inhibitoren verlängern wir die Wirkung des noch vorhandenen Acetylcholins", so Steinwachs. Auch ein Delir kann im Alter durch bestimmte Anticholinergika ausgelöst werden. Denn der ältere Mensch ist im Gegensatz zum jüngeren sehr anfällig für Delir und Wahn.

Das anticholinerge Delir ist der häufigste durch Arzneimittel hervorgerufene delirante Zustand, erklärte Steinwachs. Es gibt verschiedene periphere Symptome wie trockene Haut, Fieber, Obstipation, aber auch zentralnervöse Symptome wie Angst, Aggressivität, Halluzinationen, Verwirrtheit bis hin zu Krämpfen und Koma. Beim Delir werde zwischen hyperaktiver, hypoaktiver und gemischter Form unterschieden. „Die hypoaktiven Delire werden häufig unterschätzt. Das sind die Betroffenen, die tagsüber einfach inaktiv im Bett liegen. Dass das an einer Überdosierung von Anticholinergika liegen könnte, wird oft außer Acht gelassen", kritisierte Steinwachs.

Steinwachs rät bei der Behandlung der überaktiven Blase zentrale anticholinerge Effekte zu vermeiden. Damit ließen sich unter anderem die Zunahme von Gedächtnisstörungen im Alter, eine Veränderung des Aktivitätsmusters am Tag und in der Nacht, eine Zunahme des Acetylcholinmangels bei Demenz und eine Abschwächung der antidementiven Therapie verhindern.

Ulrike Tietze

K.-C. Steinwachs. Gehirn und Blase - Interdisziplinäre Aspekte der Therapie der $\mathrm{OAB}$ im Alter, 27.4.2013 